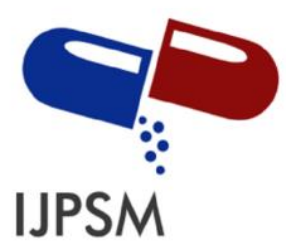

R. Senthil Prabhu et al, Int. Journal of Pharmaceutical Sciences and Medicine (IJPSM),

Vol.6 Issue. 10, October- 2021, pg. 1-15

ISSN: 2519-9889

Impact Factor: 3.426

\title{
INTERNET OF NANOTHINGS (IoNT) - A CONCISE REVIEW OF ITS HEALTHCARE APPLICATIONS AND FUTURE SCOPE IN PANDEMICS
}

\author{
*R. Senthil Prabhu ${ }^{1}$; D. Sabitha Ananthi ${ }^{1}$; S.Rajasoundarya ${ }^{2}$; R.Janakan ${ }^{3}$; R. Priyanka ${ }^{1}$ \\ ${ }^{1}$ Department of Pharmaceutics, College of Pharmacy, Madurai Medical College, Madurai-20 \\ Affiliated to The Tamilnadu Dr.M.G.R. Medical University, Chennai-32, Tamilnadu \\ ${ }^{2}$ Department of Computer science, University of Texas, El Paso, USA \\ ${ }^{3}$ Founder, Run loyal /i Trust PRO, Atlanta, Georgia, USA \\ *Corresponding Author e-mail id: grsprabhu24@yahoo.com
}

DOI: 10.47760/ijpsm.2021.v06i10.001

\begin{abstract}
Technologies that could allow literally billions of everyday objects to communicate with each other over the internet have enormous potential to change all our lives. The Internet of Things (IoT) is a transformative development, these technologies are a way of boosting productivity, keeping us healthier, making transport more efficient, reducing energy needs and making our homes more comfortable. In recent years, Internet of Things (IoT) and Internet of Nanothings (IoNT) have drawn significant research attention in numerous fields such as Healthcare, Defence, Environmental monitoring, Food and water quality control etc., There are various transformations such as Smart cities, Smart homes, Smart factories, Smart transportation, due to IoT and IoNT. Health care delivery requires the support of new technologies like IoT, IoNT to fight and look against the new pandemic diseases. For the past two years COVID-19 spreaded over worldwide including India, are fighting with pandemic disease and still looking for a practical and cost-effective solution to face the problems arising in several ways. To minimize the person to person, contact and to maintain social distancing various technologies are utilized, among them IoT and IoNT play a major role in healthcare system and allied fields. This review mainly discuss about the IoT, IoNT, its components and various applications in healthcare and allied fields.
\end{abstract}

KEYWORDS: IoT, IoNT, Nanonodes, Nano micro interface device, BSN, Drone enabled IoNT

\section{INTRODUCTION}

\subsection{Internet of Things (IoT)}

Since 1989, the birth of Internet helps us to connect Things in the internet began widely. The first Internet device, a toaster that could be turned on and off over the Internet created by John Romkey in 1990. The term IoT was coined by Kevin Ashton, executive director of the AutoID Centre, MIT in 1999 during a presentation on RFID (Radio Frequency Identification). From this point on, industrialists began to experiment more with the ability to connect devices to one another. Due to the accelerating lifestyle because of emergence of internet all of us having only limited time to check our regular routines, to govern our health and to monitor environment, the deployment of IoT is essential in all fields [1]. In the sticky current pandemic situation, the number of infected patients is escalating day by day globally, and there is a vast need to utilise the well adequate and organised facilities offered with the Internet of Things methodology. IoT has already been employed to serve the asked purposes in different domains in which the Internet of Healthcare Things (IoHT) are associated with the present issues [2]. The IoT is an emerging paradigm that enables the communication between electronic device and sensors through the internet in order to facilitate our lives. IoT use smart devices and internet to 


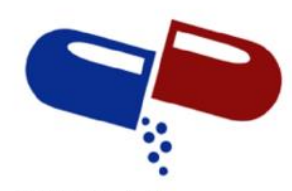

IJPSM

\section{R. Senthil Prabhu et al, Int. Journal of Pharmaceutical Sciences and Medicine (IJPSM), Vol.6 Issue. 10, October- 2021, pg. 1-15}

provide innovative solutions to various challenges and issues related to various challenges and issues related to various business, governmental and public/private industries across the world [3]. The main function of the IoT is to overcome the notch between objects in the physical world and their representation in information systems. The IoT will serve to increase the transparency and enhance the efficiency of global supply chain networks. To minimize the person to person, contact and to maintain social distancing various technologies are utilized among them IoT and IoNT play a major role in healthcare system and allied fields. IoNT enabled healthcare system is useful for proper monitoring of COVID-19 patients, by employing an interconnected network. Any physical objects can be transformed into an IoT device if it can be connected to the internet to be controlled or communicate information through a smartphone app in the hospitals and pharmaceutical industry production area [4]. The IoT is made up of Hardware and Software technologies.

i. Hardware consists of connected devices - which range from simple sensors to smart phones and wearable devices and the networks that link them, such as 4G long-term evolution, Wi-Fi and Bluetooth.

ii. Software is a data storage platforms and analytics Programmes that present information to users.

Currently $25 \%$ of the global manufacturers are using Internet of Things technologies which is anticipated to grow to over $80 \%$ by 2025 , leading to a potential global economic uplift of $\$ 2.3$ trillion in manufacturing alone [5].

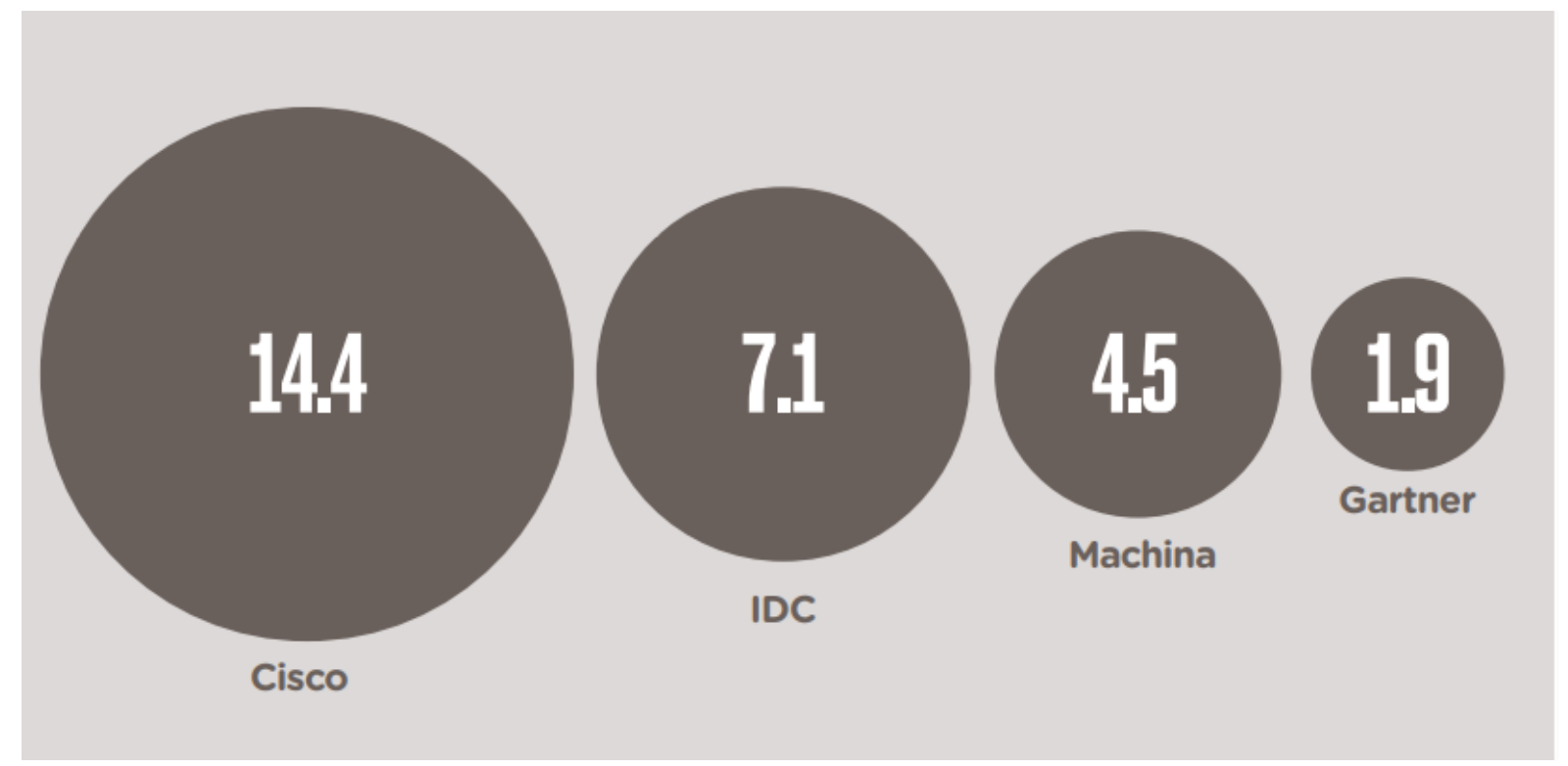

Figure 1: Global Economic Value of the Internet of Things in 2020 (\$ trillions) 


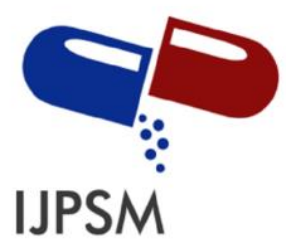

R. Senthil Prabhu et al, Int. Journal of Pharmaceutical Sciences and Medicine (IJPSM), Vol.6 Issue. 10, October- 2021, pg. 1-15

ISSN: 2519-9889

Impact Factor: 3.426

\subsection{IoT Architecture}

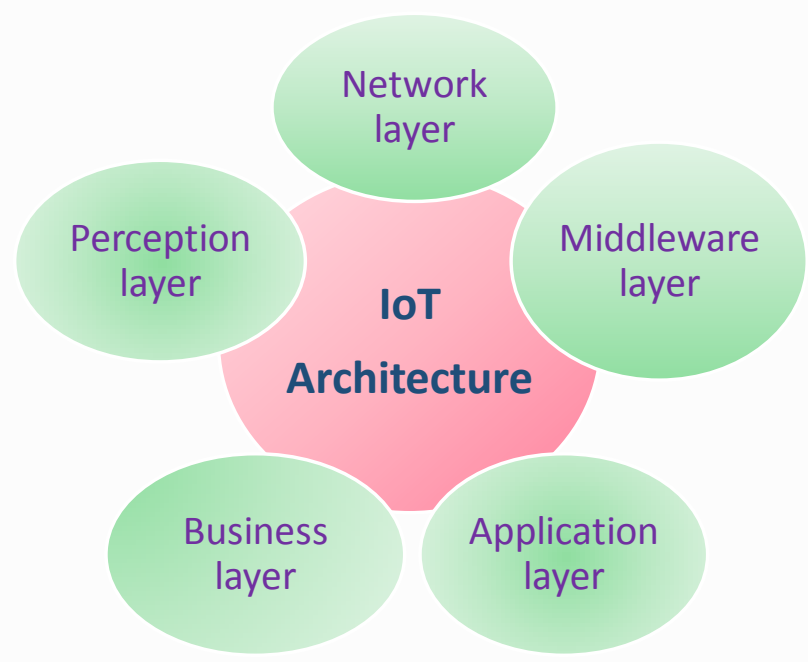

Figure 2: Five layers of IoT architecture

a) Perception layer is at the bottom of IoT architecture, consists of physical devices i.e. sensors, RFID (Radio Frequency Identification) chips, barcodes etc. and other physical objects connected in IoT network which collects information in order to deliver it to the network layer.

b) Network layer works as a transmission medium to deliver the information from perception layer to the information processing system. This transmission of information may use any wired/wireless medium along with 3G/4G, Wi-Fi, Bluetooth etc.

c) Middleware layer process the information received from the network layer and make decisions based on the results achieved from ubiquitous computing.

d) Application layer, in which processed information from the middleware layer is used for global device management.

e) Business layer is on the top of the architecture, control the overall IoT system, its applications and services. This layer visualizes the information and statistics received from the application layer and further used this knowledge to plan future targets and strategies [6].

\subsection{Internet of Nano Things (IoNT)}

IoT have shown that computers are not the only ones with a gateway to the Internet, there are various devices and objects with this access capability. It has become the most important research topic in the last ten years, whose objective is based on everyday objects having identification, detection, inter connection and processing capabilities to communicate with each other and with services through the Internet to solve a specific and useful need of people. Therefore, IoT has given researchers a thorough view of the interconnection of objects to the Internet, and with it, the IoNT has emerged. IoNT adds a new scale in IoT incorporating nanosensors in the devices, which in turn allows it to connect and communicate through the nanotechnology network with internet. These miniature sensors, interconnected through nano-networks, could obtain fine-grained data within objects and from hard-to-access areas, leading to the discovery of novel insights and applications [7]. IoNT can be defined as the interconnectivity of microscopic devices extending from one to an insufficient hundred nanometers that can access the internet and other communication networks [8]. By incorporating nano- 


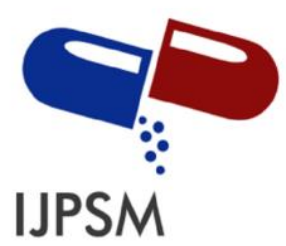

\section{R. Senthil Prabhu et al, Int. Journal of Pharmaceutical Sciences and Medicine (IJPSM), Vol.6 Issue. 10, October- 2021, pg. 1-15}

sensors in diverse objects and through the use of nano-networks IoNT is achieved [9]. The IoNT is not very different from IoT, except in the sense that devices interconnected within IoNT are miniaturized and small enough to be termed Nanoscale, which is around 1 to $100 \mathrm{~nm}$. IoNT electronics are not only sophisticated in terms of design and manufacturing, but they are extremely well-packaged to protect devices from unwanted interference. The types of nanotechnologies being integrated into an IoT system are highly specific to the application like Smart factory, connected vehicles and Body Area Network [10] [11].

\section{COMPONENTS OF IoNT}

IoNT is getting up stride in various fields. It is estimated that the market of IoNT is to grow in the upcoming future. Nano sensors of IoNT are associated with physical objects to process, gather and share information with consumers [12]. Wireless Nano sensor Networks [13] composed by several elements that are,

\subsection{Nano nodes}

Nanonodes are the nanodevices like sensors or actuators which can be installed in the person's body or in any physical network space to gather the information. These kinds of nodes are the minutest and simplest nanomachines due to having finite energy, low memory and limited communication competences due to which they can only transmit over very short distances and abilities to accomplish simple task [14]. They are diffused into a target area for sensing and collecting the information from the environment. Size of the Wireless Nano Sensor Networks (WNSN) is mainly defined by these Nanonodes because there are more in numbers to capture all relative information from the target area. The performance of the WNSN is influenced by the number of Nanonodes that are used in networks [15]. Some of the nanonodes are Biosensor, DNA sensor, and Body Sensor Network (BSN) can be included inside any person's body and also can be adjusted in different sorts of things such as books, pens, gates and so on [16].

\subsection{Nano routers}

Compared to nanonodes, nanorouters have larger computational resources which are appropriate to combine information coming from different nanomachines. A router is a key component in the internet core [17]. Nanorouters can also manage the behaviour of nanonodes by transmitting simple control commands such as sleep, on/off etc. Increasing the capabilities of these devices result in increasing their size which makes their deployment more difficult [18].

\subsection{Nano Micro Interface device}

These devices aggregate the information coming from Nanorouter and convey these information to the micro scale and vice versa. Nano-micro interfaces are the most complex hybrid devices which are able to communicate in the Nanoscale using Terahertz band and to use classical communication paradigm to communicate conventional communication networks [19].

\subsection{Gateways}

Gateways are micro scale devices that enable the remote control of the entire system over the internet. These devices can collect the information from the Nano networks and provide these information to the remotely placed monitor through the internet [20]. 


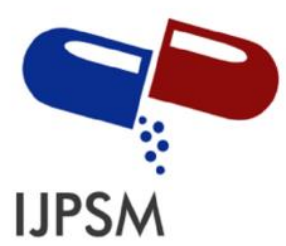

R. Senthil Prabhu et al, Int. Journal of Pharmaceutical Sciences and Medicine (IJPSM),

Vol.6 Issue. 10, October- 2021, pg. 1-15

ISSN: 2519-9889

Impact Factor: 3.426

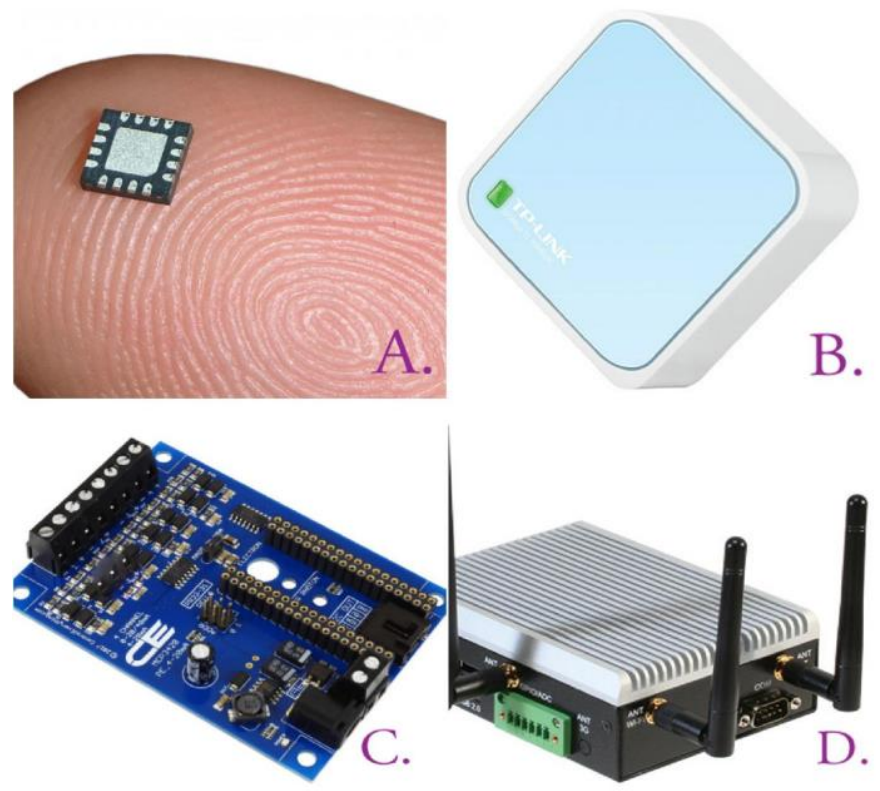

A. Nanonodes; B. Nanorouter; C. Nano micro interface device; D. Gateways

Figure 3: Components of IoNT

\section{APPLICATIONS OF IoNT}

\subsection{IONT IN HEALTH MONITORING}

The main goals of nanoscale healthcare applications are Diagnosis, Treatment, Monitoring and Prevention of diseases on the molecular, cellular, or DNA level. Current healthcare applications are implemented in two contextual domains

i. The patient's surrounding environment and

ii. The patient's body area.

The patient's daily activities can be monitored by installing sensors and actuators in a patient's surrounding environment and alert healthcare providers and emergency units if any abnormal changes in patient's health and activity. The nanonetworks can periodically sense biological properties of the tissues or organs they monitor and send readings to gateways as shown in Figure 4 [21]. Nanonetworks can also detect the presence of certain molecules, viruses and send alerts. Detection can be more enlightened and focus on abnormalities in the tissues or organs being monitored, such as indications of the onset of a heart attack. The therapeutic actions inside the body, such as administering medications, regenerative tissue engineering and nanoscale or intracellular surgery are also actively performed by Nanonetworks [22]. By leveraging IoNT, both medical professionals and patients will have access to critical healthcare data in real-time [23]. 


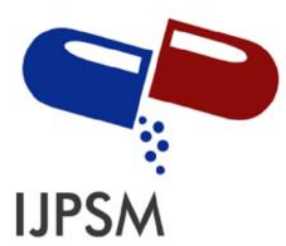

R. Senthil Prabhu et al, Int. Journal of Pharmaceutical Sciences and Medicine (IJPSM), Vol.6 Issue. 10, October- 2021, pg. 1-15

ISSN: 2519-9889

Impact Factor: 3.426

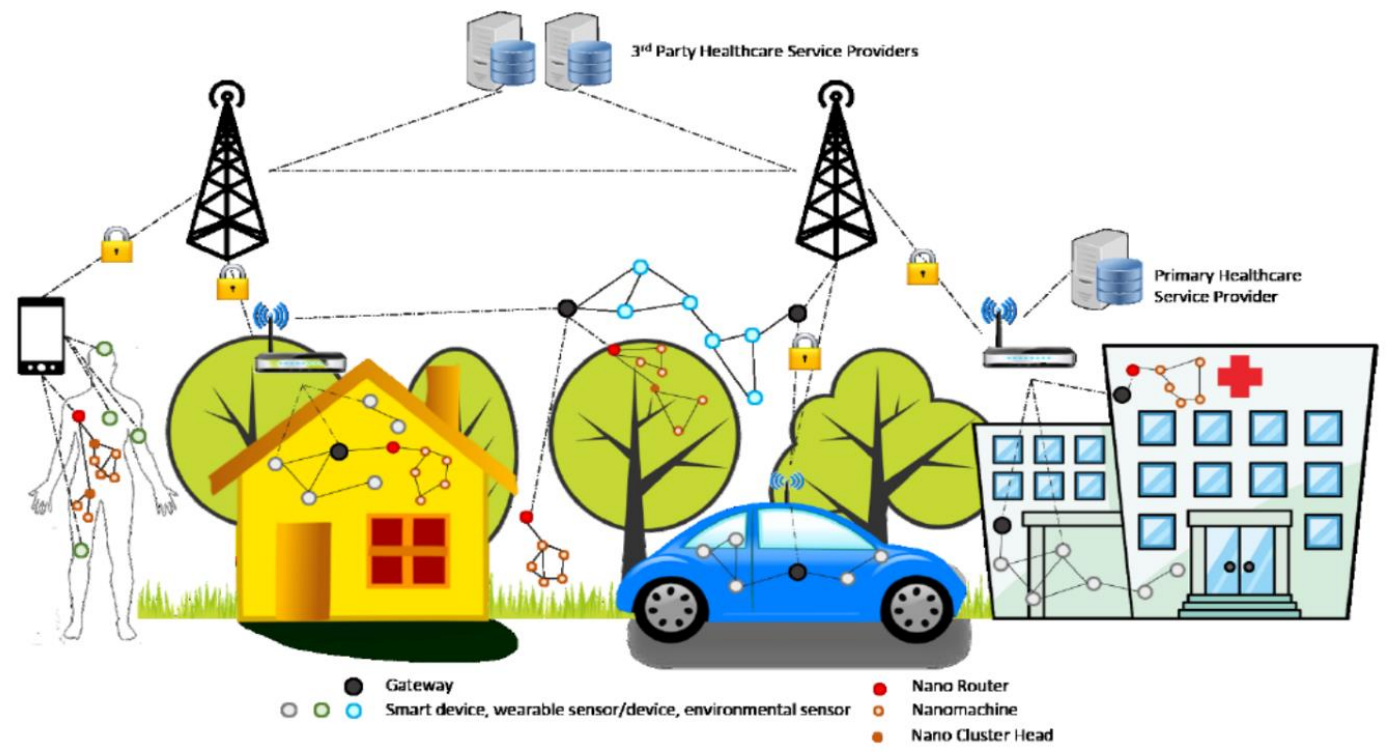

Figure 4: Generic IoNT architecture for ubiquitous healthcare

\subsubsection{Body Sensor Network (BSN)}

The IoNT often seen in recent days is BSN constituting of in-body nano sensors playing a crucial role in collecting and monitoring patient's biological activity and other details [24]. These nanosensors being used in BSN provides real time data on a wearable device being used by the doctor for getting timely information regarding patient's health [25]. Architecture of BSN communications composed of three components as shown in Figure 5,

i. Intra - BSN communication which controls and manages wearable or implanted sensors. Patient's vital signs are collected and transmitted to the sink in this tier.

ii. Inter-BSN communication in which collected information from the body is forwarded to a gateway.

iii. Beyond-BSN communication is the communication between the gateway and the doctors [26].

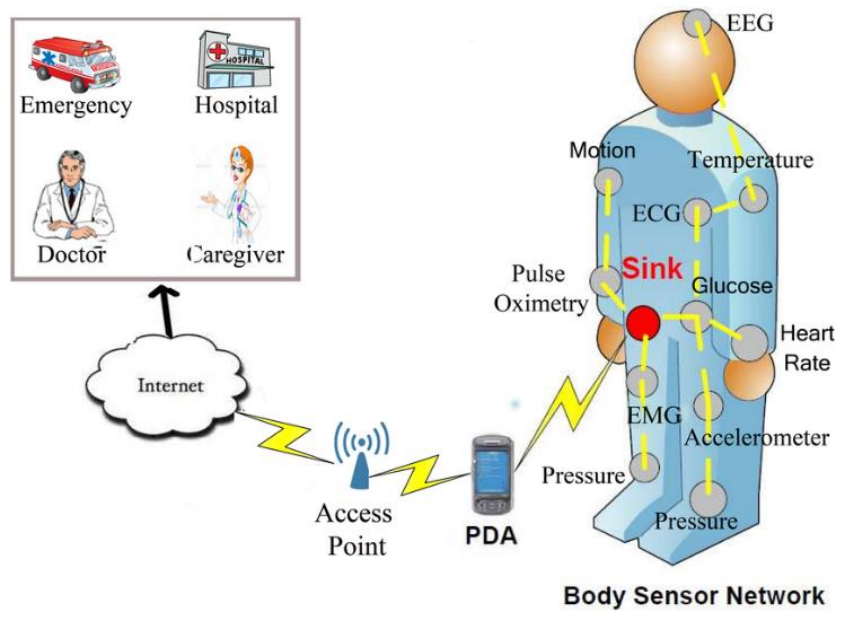

Figure 5: Body Sensor Network 


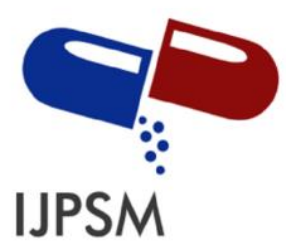

R. Senthil Prabhu et al, Int. Journal of Pharmaceutical Sciences and Medicine (IJPSM), Vol.6 Issue. 10, October- 2021, pg. 1-15

ISSN: 2519-9889

Impact Factor: 3.426

\subsubsection{Implanted Body Area Network (IBAN)}

A specific subclass of IoNT is IBAN, where nanosensors in the form of implants implanted in the human body are the Nanonodes. The IBAN encompass a small number of nanosensors communicating with the control device. It is considered to use implanted devices to assist or restore damaged neural connections with the possibility of controlling their operation from the outside [24]. The broad categories of applications of IoNT in various fields of healthcare are illustrated in Figure 6 [27].

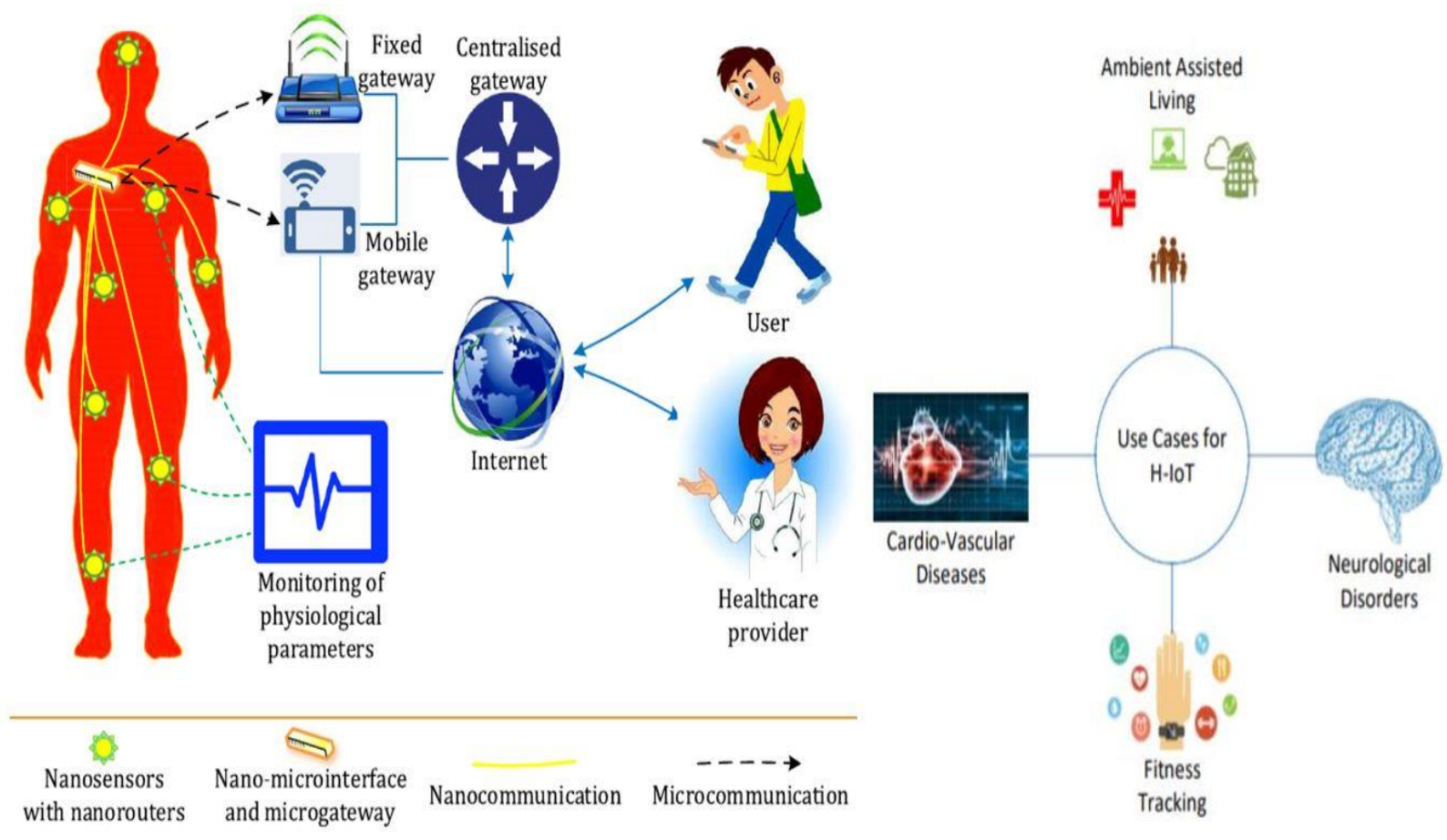

Figure 6: Broad Categories of IoNT Applications in Healthcare

\subsection{IoNT IN GENETIC ENGINEERING}

While nanothings have an artificial nature, since they are based on synthesized materials, electronic circuits and interact through Electro Magnetic (EM) communications, these characteristics can be deleterious for some application environments, such as inside the body or in natural ecosystems where the deployment of nanothings and their EM radiation could result in unwanted effects on health. By collaborating a standard IoNT and biological cell embedded computing device, a cell can be used efficiently as a substratum for the realization of the so-called Bio-Nano Things through the reuse, control and re-engineer the features of biological cells, like sensing, actuating, communication, and processing [28]. Rather than electronics, since cells are based on biological molecules and biochemical reactions, the notion of Internet of Bio-Nano thing (IoBNT) has been introduced [29]. A mapping between the components of a typical IoT embedded computing device, and the elements of a cell, becomes evident if we compare electrons' propagation in semiconductors to functionally similar, although much more complex, biochemical reactions. Elements of a biological cell and components of a typical IoNT device to control the cell functions are illustrated in Figure 7. 


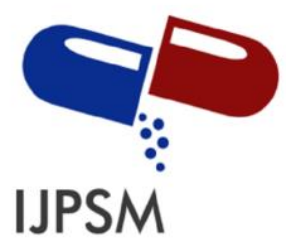

R. Senthil Prabhu et al, Int. Journal of Pharmaceutical Sciences and Medicine (IJPSM), Vol.6 Issue. 10, October- 2021, pg. 1-15

ISSN: 2519-9889

Impact Factor: 3.426

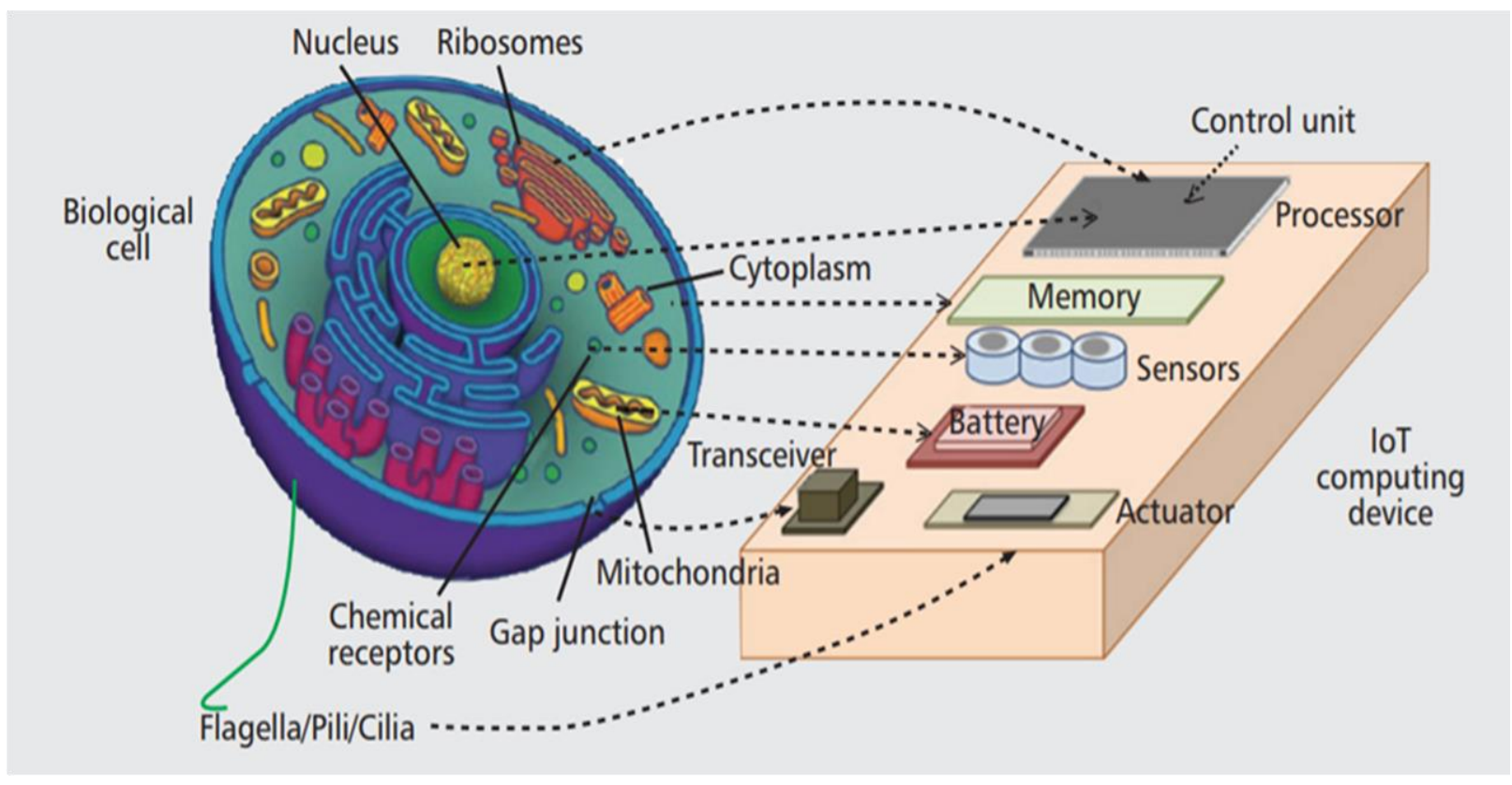

Figure 7: Elements of a biological cell and components of a typical IoNT device

i. The Control unit, which holds the embedded software of the device, would correspond to the genetic instructions densely packed into the cells' DNA molecules, which encode protein structures, the cell's data units, and regulatory sequences, similar to the software conditional expressions.

ii. The Memory unit, which accommodates the values of the embedded system data, would correspond to the chemical content of the cytoplasm, comprised of molecules synthesized by the cell as a result of DNA instructions, and other molecules or structures, e.g. vesicles, exchanged with the external environment.

iii. The Processing unit, which implements the software instructions and manages memory and peripherals, would correspond to the molecular machinery that, from the DNA molecules, through the so-called transcription and translation, generates protein molecules with instruction-dependent types and concentrations.

iv. The Power unit, which supplies the energy to maintain the electrical currents in the embedded system's circuits, would correspond to the reservoir in the cell of the Adenosine Triphosphate (ATP) molecule, which is synthesized by the cell from energy supplied from the external environment in various forms, and provides the energy necessary for the cell's biochemical reactions to occur.

v. The transceivers, which allow the embedded systems to swap the information, would correspond to the specific chains of chemical reactions, i.e., signalling pathways, through which cells exchange information-bearing molecules.

vi. Sensing and actuation, which allow embedded systems to acquire data and interact with the environment, would correspond to the ability of a cell to chemically recognize external molecules or physical stimuli, e.g., light or mechanical stress, and to change the chemical characteristics of the environment or mechanically interact through moving elements, such as flagella, pili, or cilia [30]. 


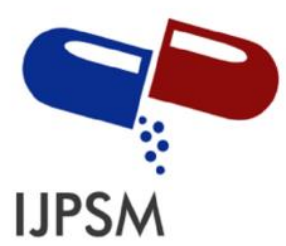

\section{R. Senthil Prabhu et al, Int. Journal of Pharmaceutical Sciences and Medicine (IJPSM), Vol.6 Issue. 10, October- 2021, pg. 1-15}

ISSN: 2519-9889

Impact Factor: 3.426

3.2.1 Thrombosis Prediction A novel IoBNT-based model with an optimized Bio-Cyber communication interface that helps in predicting and examining blood vessel clots. In this model, a bio-interface gathers the information on the blood vessels and converts it into electrical format. Further, the optical or thermal responsiveness excites the release of certain nano-carrier molecules such as liposomes which may be devised across the bloodstream and enter the targeted area passively to excite suitable nano-devices to predict the clots. The Bio-Cyber interface is used for linking the traditional electromagnetic wave to the bio-signalling network using bioluminescence concepts [28].

3.2.2 Artificial cells hold genetic information, the related molecular machineries for their transcription, translation, and replication, and all the required specialized molecules and structures. Artificial cells are expected to enable a more nimble and controllable use of synthetic biological circuits by removing all the additional complexity of natural cells that are not necessary to perform the designed functions. Although still in its infancy, this technology has been successfully applied, for drug delivery, gene therapy, and artificial blood cell production, and it is anticipated to deliver ideal substrates for synthetic biology with a more predictable behaviour [31].

\subsection{IoNT IN ENVIRONMENTAL MONITORING}

With the use of nano sensors in Environmental monitoring via deployment in public locations like Railway Stations, Bus Stops, Airports, Hotels and Restaurants and other public places, live and real time monitoring of Traffic, Air Pollution, Temperature Monitoring is done more efficiently. Some of the applications of IoNT in environmental monitoring are

3.3.1 SmartME has been one of the first initiatives in Italy to grasp a Smart City through the use of open technologies.

a) SmarME Cam is a fused hardware/software solution that can recognize and analyze objects in a generic video stream, even in real time, and may also interface with an existing network of cams. SmartMeCam lends itself to applications in challenging environments, such as airports, stations, or large public places, when it is necessary to collect statistical information about the number of people and other mobile objects, as well as their mobility patterns.

b) SmartME Parking is a service implemented in partnership with Park Smart, a start-up that aims to solve parking problems with an innovative solution based on computer vision algorithms. Park Smart proposes a solution that make use of the Edge Computing paradigm, with sensors distributed on the city and computational units that process the data and send only the parking occupancy information to the Cloud.

c) SmartME Lighting system uses conveyed waves as the main mean of communication. Each lamp is equipped with an electronic device that activates/deactivates the lamp and monitors the main consumption parameters such as voltage, current, and absorbed power [32].

\subsubsection{Vehicle tracking}

IoNT based system to gather data from vehicle sensors like rpm, speed with the help of PID

(Proportional Integral Derivative) codes from ECU (Engine Control Unit) through OBD-II (On-Board Diagnostic) to the Arduino, along with vibration and sound sensors in order to detect bottleneck with exact time taken. This data in addition with GPS was sent to cloud platform using Wi-Fi module [33]. 


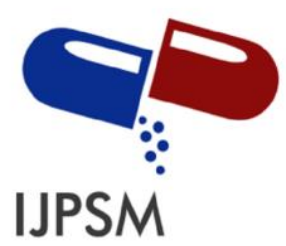

R. Senthil Prabhu et al, Int. Journal of Pharmaceutical Sciences and Medicine (IJPSM),

Vol.6 Issue. 10, October- 2021, pg. 1-15

ISSN: 2519-9889

Impact Factor: 3.426

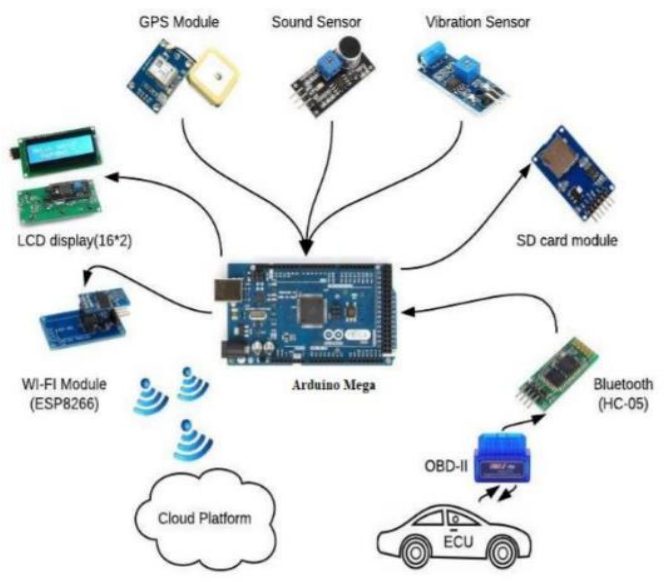

Figure 8: IoNT Application in vehicle tracking

\subsubsection{Drone enabled IoNT relay system}

Drone-enabled IoNT relay system helps to provide high-speed data collection to support remote environmental monitoring. The high-speed ability of $5 \mathrm{GHz}$ communication technology was exploited to reduce the time required for data transmission between ground monitoring devices and the drone. Long-range (LoRa) technology which functions as a low-power and long-distance wireless communication technology, is adopted as a wake-up strategy for waking up the high-power $5 \mathrm{GHz}$ module. Based on $5 \mathrm{GHz}$ and LoRa technology, a drone-based on-board relay and ground intelligent terminal are designed [34].

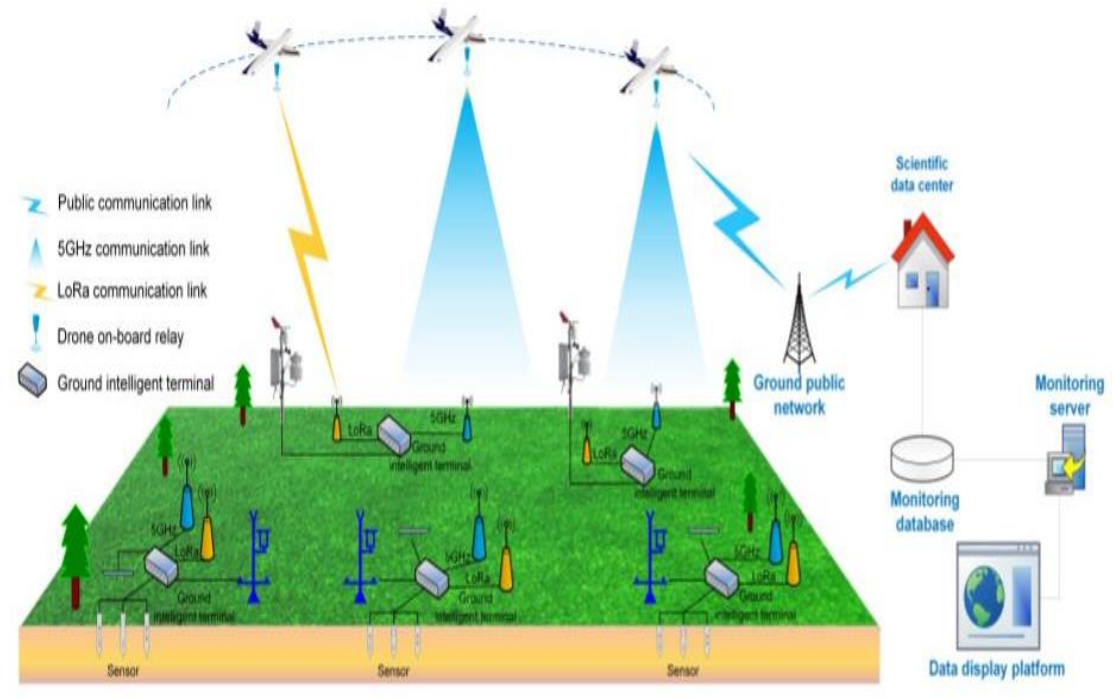

Figure 9: Drone-enabled IoNT relay system

\subsection{IoNT IN NUCLEAR BIOLOGICAL AND CHEMICAL (NBC) DEFENCE}

Nanosensors and Nanoactuators are deployed to detect aggressive chemical and biological agents over the battlefield or targeted areas. Nanosensor networks can be used to detect the unauthorized entrance of 


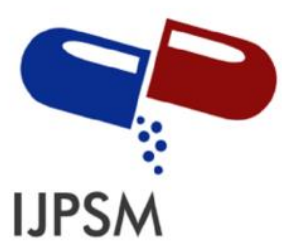

R. Senthil Prabhu et al, Int. Journal of Pharmaceutical Sciences and Medicine (IJPSM), Vol.6 Issue. 10, October- 2021, pg. 1-15

ISSN: 2519-9889

Impact Factor: 3.426

Cargo containers containing biological, chemical, and radiological materials. IoNT is envisaged to benefit two types of military applications Nuclear and bio-chemical detection and Physical damage detection [35].

\subsubsection{Promising target scenarios for defence and public safety}

a) C4ISR (Command, Control, Communications, Computers, Intelligence, Surveillance, and Reconnaissance)

Millions of sensors used by C4ISR systems are deployed on a range of platforms to provide advanced situational awareness. Radar, video, infrared or passive radio frequency detection data are gathered by surveillance satellites, airborne platforms, UAVs (Unmanned Aerial Vehicles), ground stations, and soldiers in the field. These data are delivered to an integration platform that analyses them and delivers information up and down the chain of command. These platforms provide a Common Operational Picture (COP) allowing for enhanced coordination and control across the field. High-level military echelons are provided with comprehensive situational awareness through central operations centres, which receive data feeds from platforms. Lower levels (i.e., platoon, soldiers) also have access to the data in their area. In the case of combat pilots, they receive prioritized data feeds integrated with data from their own sensor systems [36].

\section{b) Surveillance}

Security cameras and sensors, amalgamated with sophisticated image analysis and pattern recognition software, ease remote facility monitoring for security threats. For marine and coastal surveillance, using different kinds of sensors integrated in planes, unmanned aerial vehicles, satellites and ships, make possible to control the maritime activities and traffic in large areas, keep track of fishing boats, and supervise environmental conditions and dangerous oil cargos. Monitoring of hazardous situations such as combustion gases and pre-emptive fire conditions to define alert zones, monitoring of soil moisture, vibrations and earth density measurements to detect dangerous patterns in land conditions or earthquakes, or distributed measurement of radiation levels in the surroundings of nuclear power stations to generate leakage alerts [36].

\section{c) Personal Sensing, Soldier Healthcare and Workforce Training}

Body-worn devices [37] are increasingly used by soldiers [38]. Fitness trackers [39] enable monitoring of physical activity along with vital signs. This information has an obvious value for the users, but there is also a significant potential in examining aggregate values of communities. Body-worn sensors, when deployed on a community scale, offer information to support C4ISR. Technologies for monitoring both workforce and their surroundings could aid when inferring physical or psychological states as well as assessing the risk of internal injury based on prior trauma [40]. Soldiers can be alerted of abnormal states such as dehydration, sleep deprivation, elevated heart rate or low blood sugar and, if necessary, warn a medical response team in a base hospital. These wide range of health and security monitoring systems, enables an effective endto-end soldier health system, including re-provisioning of health services when needed.

\subsection{IONT IN FOOD AND WATER QUALITY CONTROL}

The use of IoNT has the potential to escort the development of several precision farming applications. Accordingly, design of nano-devices (nano-sensors and nanoactuators) has been proposed in order to track environment variables, soil fertility, crop growth and animal health at the nano-scale [41].

\subsubsection{Grass monitoring}

a. Nanodevices are used to closely monitor the dynamics between plant cell organelles and pathogens to permit early prediction and prevention of diseases.

b. Nanosensors are used to monitor the soil fertility and

c. Nanoscale actuators designed to allow controlled delivery of fertilizers to the soil, thereby, preventing soil depletion. 


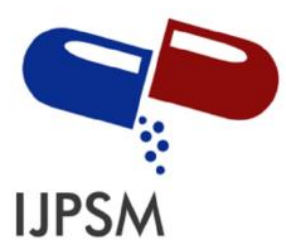

R. Senthil Prabhu et al, Int. Journal of Pharmaceutical Sciences and Medicine (IJPSM), Vol.6 Issue. 10, October- 2021, pg. 1-15

ISSN: 2519-9889

Impact Factor: 3.426

Dynamic irrigation can be practised by observing the soil moisture levels in real-time, to avoid water-logging and wastage [42].

\subsubsection{Animal health and feed management}

Nano-scale drug delivery systems shall allow controlled release of medication to specific sites in order to alleviate the spread of diseases. Nano-sensors to monitor hormone levels in the cattle, for improved fertility, may also be designed. Additionally, nanoscale carriers may be used to improve the nutrient profile of the feedstock. Feeding efficiency may be further improved by adding nano-scale digestive-aids.

\subsubsection{Water quality control}

Water distribution for the household use and agriculture, incorporation of network would consist of an integrated array of nanosensors that would provide real-time monitoring of water quantity and quality both at fine-grained temporal and geographic scales and at locations throughout the distribution system that are inaccessible to existing approaches (dead-end pipes, connections between service mains, service lines and premise plumbing, within biofilms, in showerheads) [43].

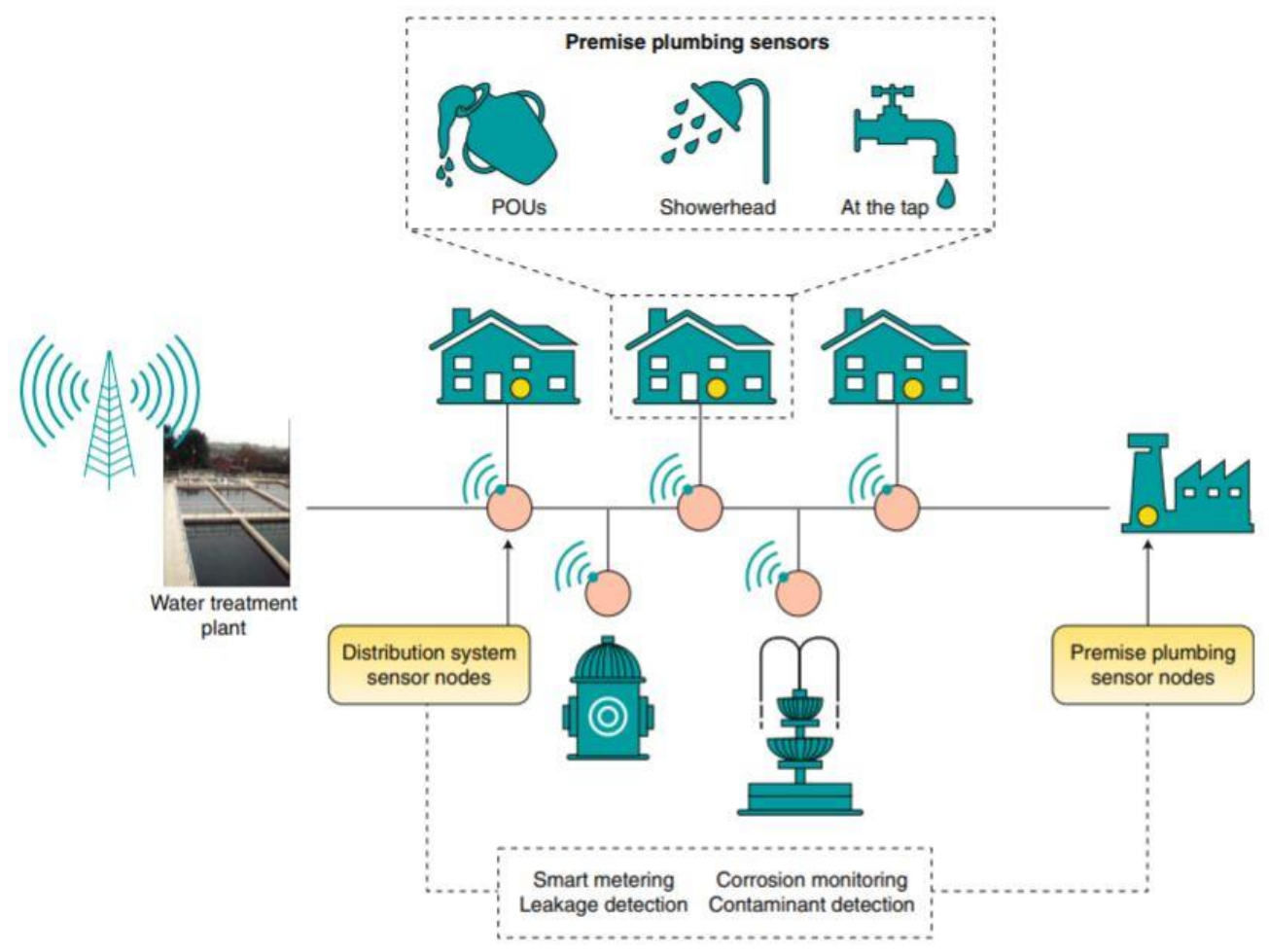

Figure 10: Internet of NanoThings (IoT)-enabled water sensing

\subsubsection{Food safety monitoring}

FSSAI has established a regulatory standard for microbial and non-microbial contaminants in milk and milk products. Therefore, dairy industries or food business operators (FBOs) are needed to follow these standards during the manufacture, marketing, retails, and distribution of various dairy products._Some of the applications of IoNT in food safety monitoring are, 


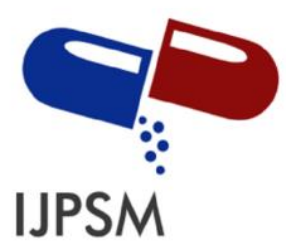

R. Senthil Prabhu et al, Int. Journal of Pharmaceutical Sciences and Medicine (IJPSM), Vol.6 Issue. 10, October- 2021, pg. 1-15

ISSN: 2519-9889

Impact Factor: 3.426

a. The nanobiosensors are developed by using specific recognition molecules which are integrated on a surface of the nanowire/nanotube for making a specifically sensitive to the target. The Wide spectrum of recognition molecule like single-stranded DNA, an antibody, aptamer, enzymes, protein which shows an affinity toward a target, or a protein that specifically interact with another biological molecule. These nanobiosensors are having wide application in the field of microbial quality and safety monitoring in dairy industry including antibiotics, pesticides, heavy metals, aflatoxin, and adulterants, microbial contamination including foodborne pathogens, and packaging material integration with nanobiosensor as an indicator of quality and safety of the products.

b. The development of lab-on-a-chip technique by integration of analyte onto a microfluidic chip to develop an electromechanical system, would provide new avenue field of nanobiosensor [44].

\section{FUTURE PERSPECTIVE OF IONT}

The Internet of Nano Things Market was valued at USD 1,761.57 million in 2020 and is expected to reach USD 11,148.62 million by 2026, register a CAGR (Compound Annual Growth Rate) of $35.08 \%$ over the forecast period (2021 - 2026). During the COVID-19 pandemic, this IoNT technology has shown very encouraging results dealing with this disease. IoT technology during this pandemic has proven its usefulness in assisting patients, healthcare providers, and authorities. Many smartphone applications have been developed and some works are in progress for the healthcare domain, and some of them have been used in response to COVID-19, namely nCapp, DetectaChem, Stop Corona, Social Monitoring, Civitas, StayHomeSafe, AarogyaSetu, TraceTogether, Hamagen, Coalition, BeAware Bahrain, eRouska. Autonomous/Driverless vehicle technology, Smart city, Electrical Grid Industry are some of the fields which are the future research areas to ameliorate the IoNT technology [45]. Some of the top companies [46] in IoT and IoNT industries are,

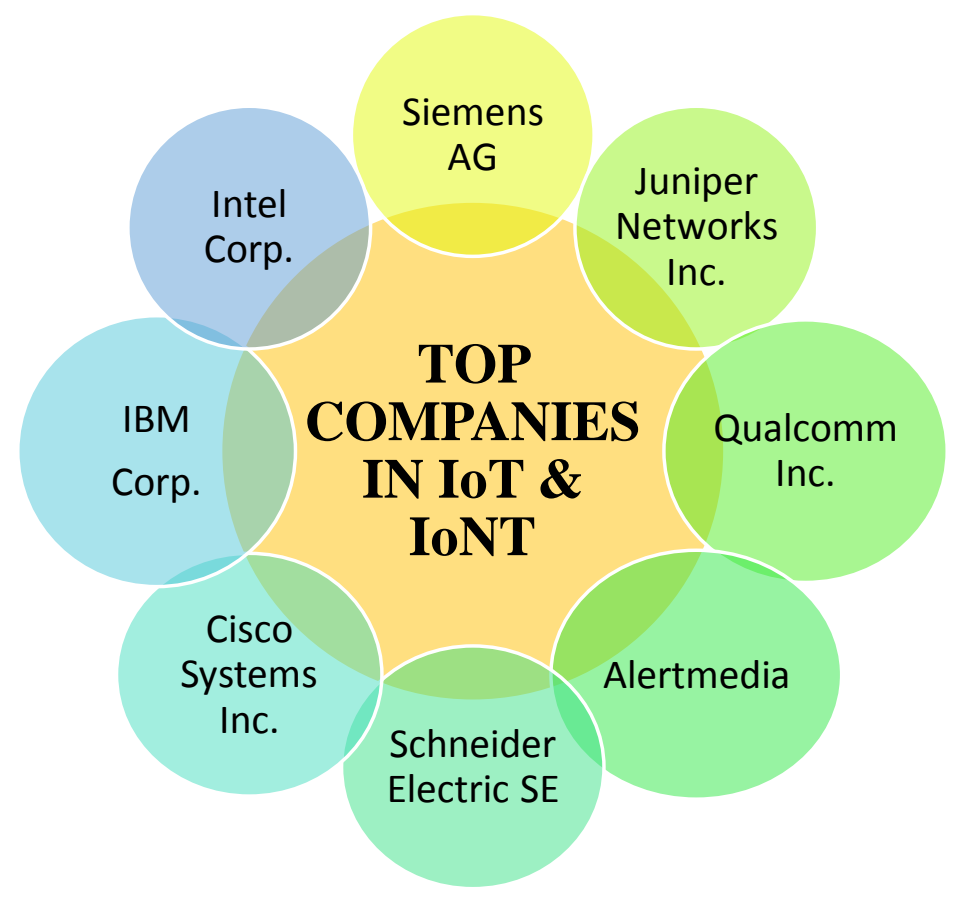

Figure 11: Top Companies in IoT and IoNT 


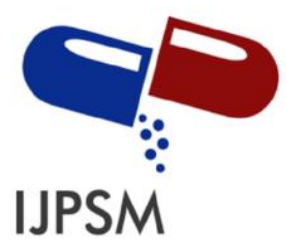

\section{R. Senthil Prabhu et al, Int. Journal of Pharmaceutical Sciences and Medicine (IJPSM), Vol.6 Issue. 10, October- 2021, pg. 1-15}

ISSN: 2519-9889

Impact Factor: 3.426

\section{CONCLUSION}

This paper has summarized the emerging areas of specific IoNT technologies and has given the basic components present in IoNT. The IoNT holds the promise of improving people's lives through both automation and augmentation. The capabilities offered by the IoNT can save people and organizations time and money as well as help improve decision making and outcomes in a wide range of application areas. The most acute challenges that the IoNT paradigm pose for healthcare applications and services are the design of efficient information dissemination and routing schemes, and the accurate channel and signal propagation modelling for intra-body communication. As seen from today's perspective, it seems to be real that in a few years implanting health sensors and using the IoT and IoNT networks in order to manage people's health will be just as popular as vaccination. A bigger challenge is the seamless integration of IoNT with current health-based IoNT systems and networks, and the efficient and dynamic orchestration of health services across the heterogeneous networking and data governance domains.

\section{REFERENCES}

[1]. Suresh P. Daniel JV. Parthasarathy V. Aswathy RH, 2014 Nov 27, A state of the art review on the Internet of Things (IoT) history, technology and fields of deployment. In2014 International conference on science engineering and management research (ICSEMR), IEEE., 1-8.

[2]. Singh RP. Javaid M. Haleem A. Suman R, 2020 Jul 1, Internet of things (IoT) applications to fight against COVID-19 pandemic. Diabetes \& Metabolic Syndrome: Clinical Research \& Reviews, 14(4), 521-524.

[3]. Sfar AR. Chtourou Z. Challal Y, 2017 Feb 17, A systemic and cognitive vision for IoT security: a case study of military live simulation and security challenges. In2017 International Conference on Smart, Monitored and Controlled Cities (SM2C) IEEE, 101-105.

[4]. Weber RH. Weber R, 2010, Internet of things-Legal perspectives, Springer, 1-22.

[5]. Sir Mark Walport - UK Government Chief Scientific Adviser, 2014, The Internet of Things: making the most of the Second Digital Revolution | Oxford Martin School, 13-20.

[6]. Kumar S. Tiwari P. Zymbler M, 2019 Dec, Internet of Things is a revolutionary approach for future technology enhancement: a review, Journal of Big Data, 6(1), 1-21.

[7]. Cruz Alvarado MA. Bazán P, 2019 Jun, Understanding the Internet of Nano Things: overview, trends, and challenges, ECiencias de la Información, 9(1), 152-182.

[8]. Rafae H, Jamil SW. Aslam MI. Ahmed I, 2019, Internet of nano Things: Next step for future of nanotechnology, InProc. 4th International Electrical Engineering Conference (IEEC), (p. 8).

[9]. Miraz MH. Ali M. Excell PS. Picking R, 2015 Sep 8, A review on Internet of Things (IoT), Internet of everything (IoE) and Internet of nano things (IoNT), In2015 Internet Technologies and Applications (ITA), IEEE, 219-224.

[10]. Dressler F. Fischer S, 2015 Jun 1, Connecting in-body nano communication with body area networks: Challenges and opportunities of the Internet of Nano Things, Nano Communication Networks, 6(2), 29-38.

[11].Akyildiz IF. Jornet JM, 2010 Dec 23, The internet of nano-things, IEEE Wireless Communications, 17(6), 58-63.

[12]. Ealia SA. Saravanakumar MP, 2017 Nov 1, A review on the classification, characterisation, synthesis of nanoparticles and their application, InIOP Conference Series: Materials Science and Engineering, Vol. 263, No. 3, p.032019.

[13]. Rupani V. Kargathara S. Sureja J, 2015, A review on wireless nanosensor networks based on electromagnetic communication, International Journal of Computer Science and Information Technologies, 6(2), 1019-1022.

[14]. Dressler F. Kargl F, 2012 Sep 1, Towards security in nano-communication: Challenges and opportunities, Nano communication networks, 3(3), 151-160.

[15].Piro G. Grieco LA. Boggia G. Camarda P, 2013 Mar 25, Simulating wireless nano sensor networks in the ns-3 platform, In2013 27th International Conference on Advanced Information Networking and Applications Workshops, (pp. 67-74).

[16]. Akyildiz IF. Jornet JM, 2010 Mar 1, Electromagnetic wireless nanosensor networks, Nano Communication Networks, 1(1), 3-19.

[17]. Sardinha LH. Costa AM. Neto OP. Vieira LF. Vieira MA, 2013 Dec, Nano router: a quantum-dot cellular automata design, IEEE Journal on Selected Areas in Communications, 31(12), 825-834.

[18]. Nayyar A. Puri V. Le DN, 2017 Jul, Internet of nano things (IoNT): Next evolutionary step in nanotechnology, Nanoscience and Nanotechnology, 7(1), 4-8.

[19].Akkari N. Wang P. Jornet JM. Fadel E. Elrefaei L. Malik MG. Almasri S. Akyildiz IF, 2016 May 26, Distributed timely throughput optimal scheduling for the Internet of nano-things, IEEE Internet of Things Journal, 3(6), 1202-1212. 


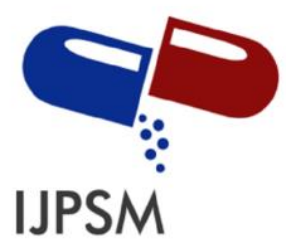

\section{R. Senthil Prabhu et al, Int. Journal of Pharmaceutical Sciences and Medicine (IJPSM), Vol.6 Issue. 10, October- 2021, pg. 1-15}

[20].Piro G. Grieco LA. Boggia G. Camarda P, 2013 Mar 5, Nano-Sim: simulating electromagnetic-based nanonetworks in the network simulator 3, InSimuTools, 203-210.

[21].Ali NA. Abu-Elkheir M, 2015 Oct 19, Internet of nano-things healthcare applications: Requirements, opportunities, and challenges, In2015 IEEE 11th International Conference on Wireless and Mobile Computing, Networking and Communications (WiMob), (pp. 9-14).

[22]. Gopinath SC. Tang TH. Chen Y. Citartan M. Lakshmipriya T, 2014 Oct 15, Bacterial detection: From microscope to smartphone. Biosensors and Bioelectronics, 60, 332-342.

[23].El-Din HE. Manjaiah DH, 2017, Internet of nano things and industrial internet of things, InInternet of Things: Novel Advances and Envisioned Applications, Springer, (pp. 109-123).

[24].Jarmakiewicz J. Parobczak K. Maślanka K, 2016 May 23, On the Internet of Nano Things in healthcare network, In2016 International Conference on Military Communications and Information Systems (ICMCIS), IEEE, (pp. 1-6).

[25]. Balasubramaniam S. Kangasharju J, 2012 Nov 20, Realizing the internet of nano things: challenges, solutions, and applications, Computer, 46(2), 62-68.

[26]. Ayatollahitafti V. Ngadi MA. Mohamad Sharif JB. Abdullahi M, 2016 Jan 15, An efficient next hop selection algorithm for multi-hop body area networks, PloS one, 11(1), e0146464.

[27].Qadri YA. Nauman A. Zikria YB. Vasilakos AV. Kim SW, 2020 Feb 11, The future of healthcare internet of things: a survey of emerging technologies, IEEE Communications Surveys \& Tutorials, 22(2), 1121-1167.

[28].Fouad H. Hashem M. Youssef AE, 2020 Jul, A Nano-biosensors model with optimized bio-cyber communication system based on Internet of Bio-Nano Things for thrombosis prediction, Journal of Nanoparticle Research, 22(7), 1-7.

[29].Akyildiz IF. Brunetti F. Blazquez C, 2008 Aug 22, Nanonetworks: A new communication paradigm, Computer Networks, 52(12), 2260-2279.

[30].Akyildiz IF. Pierobon M. Balasubramaniam S. Koucheryavy Y, 2015 Mar 18, The internet of bio-nano things, IEEE Communications Magazine, 53(3), 32-40.

[31].Wu F. Tan C, 2014 Jul, The engineering of artificial cellular nano systems using synthetic biology approaches, Wiley Interdisciplinary Reviews: Nanomedicine and Nanobiotechnology, 6(4), 369-83.

[32]. Bruneo D. Distefano S. Giacobbe M. Minnolo AL. Longo F. Merlino G. Mulfari D. Panarello A. Patanè G. Puliafito A. Puliafito C, 2019 Mar 1, An iot service ecosystem for smart cities: The\# smartme project, Internet of Things, 5, 12-33.

[33].Zeb A. Agha A. Ayaz S. Ullah MR. Ullah M, 2020 Nov, IoT based system for spotting bottleneck and its cost as a time wastage through OBD II, International Research Journal of Modernization in Engineering Technology and Science, 596601.

[34].Zhang M. Li X, 2020 Apr 16, Drone-enabled Internet-of-Things relay for environmental monitoring in remote areas without public networks, IEEE Internet of Things Journal, 7(8), 7648-7662.

[35]. Yu H, 2018, Data Acquisition and Dissemination for the Internet of Nano Things, 21-25.

[36]. Fraga-Lamas P. Fernández-Carames TM. Suarez-Albela M. Castedo L. Gonzalez-Lopez M, 2016 Oct, A review on internet of things for defense and public safety, Sensors, 16(10), 1644.

[37].Cirani S. Picone M, 2015 Sep 21, Wearable computing for the internet of things. It Professional, 17(5), 35-41.

[38]. Catherwood PA. Finlay DD. McLaughlin JA, 2015 Nov 11, Subcutaneous body area networks: A SWOT analysis, In2015 IEEE International Symposium on Technology and Society (ISTAS), IEEE, (pp. 1-8).

[39]. Alderson A, 2015 May 1, Sports Tech: Fitness Trackers. Engineering \& Technology, 10(4), 84-85.

[40]. Shunk D, 2015 Jan 1, Ethics and the enhanced soldier of the near future. Military Review, 95(1), 91.

[41]. Bhargava K. Ivanov S. Donnelly W, 2015 Sep 21, Internet of nano things for dairy farming, In Proceedings of the Second Annual International Conference on Nanoscale Computing and Communication, 1-2.

[42]. Chen H. Yada R, 2011 Nov 1, Nanotechnologies in agriculture: new tools for sustainable development, Trends in Food Science \& Technology, 22(11), 585-594.

[43].Vikesland PJ, 2018 Aug, Nanosensors for water quality monitoring. Nature nanotechnology, 13(8), 651-60.

[44]. Raghu HV. Parkunan T. Kumar N, 2020 Jan 02, Application of nanobiosensors for food safety monitoring, Environmental Nanotechnology, Springer, Volume 4: (pp. 93-129).

[45].Nasajpour M. Pouriyeh S. Parizi RM. Dorodchi M. Valero M. Arabnia HR, 2020 Nov 12, Internet of Things for current COVID-19 and future pandemics: An exploratory study, Journal of healthcare informatics research, 1-40.

[46].Riadi S. Triono S. Syahril S. Nofriansyah D, 2019, Effectiveness of Metcaognitive Learning's Model in Engineering, International Journal of Engineering and Advanced Technology (IJEAT), 9(1), 4438-4443. 